\title{
Synthetic satellite imagery in the Lokal-Modell
}

\author{
Christian Keil ${ }^{\mathrm{a}, *}$, Arnold Tafferner ${ }^{\mathrm{a}}$, Thorsten Reinhardt ${ }^{\mathrm{b}}$ \\ a Institut für Physik der Atmosphäre, DLR Oberpfaffenhofen, Germany \\ ${ }^{\mathrm{b}}$ Deutscher Wetterdienst, Offenbach, Germany
}

Received 19 January 2005; accepted 15 January 2006

\begin{abstract}
The implementation of a forward radiative transfer model into the code of the Lokal-Modell (LM) of Deutscher Wetterdienst (DWD) allows for the operational computation of synthetic satellite imagery. This new diagnostic tool (LMSynSat, Lokal-Modell Synthetic Satellite imagery) is used to evaluate forecast quality using observed satellite data of Meteosat 7 and Meteosat 8 . An obvious model deficiency in simulating high clouds can be overcome by running the LM with new prognostic precipitation and cloud-ice schemes providing more realistic cloud properties. Additionally, the inclusion of precipitating snow crystals in the computation of brightness temperature achieves better agreement of upper tropospheric clouds. Aiming at an objective usage of satellite imagery to select good members out of an ensemble of LM forecasts requires a realistic representation of clouds in the LM. (C) 2006 Elsevier B.V. All rights reserved.
\end{abstract}

Keywords: Mesoscale model; Meteosat; Model validation; Clouds

\section{Introduction}

Remote sensing observations from satellite and radar represent rich sources of data that can be potentially used to improve weather forecasts through data assimilation or to evaluate forecast quality. However, a direct comparison between observation and model forecast, regarding for example the occurrence of clouds, is complicated because satellites measure brightness temperature (BT in $\mathrm{K}$ ), a quantity that is not forecast by the Lokal-Modell (LM). One method is the 'observation-to-model approach' or 'retrieval', in which a quantity comparable to the model-predicted quantity is derived from remotely sensed data. For

\footnotetext{
* Corresponding author. Deutsches Zentrum für Luft-und Raumfahrt, Institut für Physik der Atmosphäre, Oberpfaffenhofen, 82234 Wessling, Germany.

E-mail address: christian.keil@dlr.de (C. Keil).
}

satellite measurements, complex algorithms are used to retrieve the cloud information (e.g. Rossow and Schiffer, 1991; Stubenrauch et al., 1999). Such derived products may be ambiguous due to uncertainties arising from the background guess required to convert radiances to temperature profiles. The guess profiles usually contain biases from the forecast model that propagate into the retrieval (Chevallier et al., 2001). On the other hand, a forward radiative transfer model can be used to simulate measurements obtained by remote sensing instruments from the forecast model fields. Such a 'model-to-observation approach' allows direct comparison between observed and simulated satellite images and can be used to evaluate forecast quality. Recent progress in constructing synthetic satellite images from model output makes such an approach feasible (Morcrette, 1991; Schmetz and van de Berg, 1994; Chaboureau et al., 2000; Chevallier and Kelly, 2002). Here, the 'model-to-observation approach' is followed 
to calculate synthetic satellite images from forecast model fields.

In the next section, the mesoscale model and observational data are presented. This is followed by a section devoted to the generation of synthetic satellite imagery. In Section 4, the case-study results are presented and discussed. Finally, the work is summarized and an outlook is given using synthetic satellite imagery in the context of a regional ensemble system.

\section{Model and observational data}

The non-hydrostatic Lokal-Modell (LM) (Doms and Schättler, 1999; Steppeler et al., 2003) has been the operational short range weather forecasting tool at Deutscher Wetterdienst (DWD) since December 1999. In the operational configuration with a horizontal mesh size of $7 \mathrm{~km}$, the model domain encompasses all of Central Europe. The resolution will be increased to $2.8 \mathrm{~km}$ in 2006 (Doms and Förstner, 2004). The LM vertical coordinate is of a generalized terrain-following type, which divides the atmosphere in 35 layers from the bottom up to $20 \mathrm{hPa}$. The prognostic variables are the three wind components, temperature, pressure perturbation, specific humidity and cloud liquid water. The model physics include a level-2 turbulence parameterization, a delta-2-stream radiation scheme and a two-layer soil model. The model includes a grid-scale cloud and precipitation scheme as well as a parameterization of moist convection (Tiedtke, 1989). A cloud-ice scheme has been introduced in the gridscale precipitation parameterization adding cloud ice as a new prognostic variable in 2003 (Doms et al., 2004). Recently, prognostic precipitation using a threedimensional semi-Lagrangian advection scheme has been implemented in LM with additional prognostic variables for snow and rain content (Baldauf and Schulz, 2004).

Satellite data from the geostationary satellite Meteosat 7 is used in the present study. Meteosat 7 is currently positioned at the equator on the Greenwich meridian. The infrared window channel (IR, $10.1-13.0 \mu \mathrm{m})$ is sensitive to cloud amount throughout the troposphere, whereas the water vapor absorption band (WV, 5.4-7.6 $\mu \mathrm{m}$ ) primarily detects the water vapor content in the upper troposphere. The uncertainty caused by calibration error amounts to $2-$ $4 \mathrm{~K}$ (Köpken, 2001) and is significantly smaller than the cloud signals investigated in the present study. The satellite's spatial resolution is $5 \mathrm{~km}$ at nadir and about $8 \mathrm{~km}$ at $50^{\circ} \mathrm{N}$, comparable to the current resolution of the LM.

\section{Generation of synthetic satellite imagery}

Synthetic satellite imagery is generated using the fast radiative transfer model for TIROS Operational Vertical Sounder (RTTOV), originally developed at European Centre for Medium Range Weather Forecast (ECMWF) in the early 1990s (Eyre, 1991). Subsequently, the original code has gone through several developments (Saunders et al., 1999; Chevallier et al., 2001; Chevallier and Kelly, 2002; Matricardi et al., 2001), more recently within the EUMETSAT NWP Satellite Application Facility (SAF). RTTOV allows fast simulation of brightness temperatures for satellite infrared or microwave radiometers given an atmospheric profile of temperature, variable gas concentrations, cloud and surface properties. RTTOV plays a key role in the assimilation of satellite data, for instance at ECMWF.

The forward calculation necessary to generate synthetic brightness temperature has been implemented as a diagnostic tool into the LM (Keil and Tafferner, 2003). The LM fields used to compute synthetic brightness temperature are specific humidity, cloud liquid water, cloud ice, cloud cover, skin temperature, specific humidity and temperature at $2 \mathrm{~m}$, and land-sea mask (see Table 1). Using the new prognostic precipitation scheme enables the additional use of water content of snow. Because a snow category (distinct from cloud ice) does not exist in RTTOV, the sum of snow and cloud ice (instead of cloud ice alone) is used to compute brightness temperature. In RTTOV, the radius of cloud ice is assumed to vary between 30 and $60 \mu \mathrm{m}$ (Chevallier et al., 2001). Since snow particles are larger $(>100 \mu \mathrm{m})$ and, therefore, for a given mass concentration, the emissivity of snow is lower than that of cloud ice, the impact of snow is overestimated in the RTTOV calculations. However, a good improvement of the high cloudiness in the synthetic satellite images is

Table 1

List of LM input variables

\begin{tabular}{llll}
\hline Variable & Name & Units & Dimension \\
\hline Temperature & $\mathrm{T}$ & $\mathrm{K}$ & 3 \\
Specific humidity & $\mathrm{qv}$ & $\mathrm{kg} / \mathrm{kg}$ & 3 \\
Cloud liquid water & $\mathrm{qc}$ & $\mathrm{kg} / \mathrm{kg}$ & 3 \\
Cloud ice water & $\mathrm{qi}$ & $\mathrm{kg} / \mathrm{kg}$ & 3 \\
Water content of snow & $\mathrm{qs}$ & $\mathrm{kg} / \mathrm{kg}$ & 3 \\
Cloud cover & $\mathrm{clc}$ & $0-1$ & 3 \\
Pressure & $\mathrm{p}$ & $\mathrm{Pa}$ & 3 \\
Surface pressure & $\mathrm{ps}$ & $\mathrm{Pa}$ & 2 \\
Skin temperature & $\mathrm{T} \_\mathrm{g}$ & $\mathrm{K}$ & 2 \\
2-m temperature & $\mathrm{T} \_2 \mathrm{~m}$ & $\mathrm{~K}$ & 2 \\
2-m specific humidity & $\mathrm{qv}$-2m & $\mathrm{kg} / \mathrm{kg}$ & 2 \\
Land-sea mask & fr_land & $0-1$ & 2 \\
\hline
\end{tabular}


Table 2

List of output variables: for every channel/wavelength clear and cloudy-sky radiances and brightness temperatures are archived

\begin{tabular}{llcl}
\hline Satellite & Sensor & Channel & Central wavelength \\
\hline Meteosat 7 & MVIRI & 1 & WV6.4 \\
Meteosat 7 & MVIRI & 2 & IR11.5 \\
Meteosat 8 & SEVIRI & 4 & IR3.9 \\
Meteosat 8 & SEVIRI & 5 & WV6.2 \\
Meteosat 8 & SEVIRI & 6 & WV7.3 \\
Meteosat 8 & SEVIRI & 7 & IR8.7 \\
Meteosat 8 & SEVIRI & 8 & IR9.7 \\
Meteosat 8 & SEVIRI & 9 & IR10.8 \\
Meteosat 8 & SEVIRI & 10 & IR12.1 \\
Meteosat 8 & SEVIRI & 11 & IR13.4 \\
\hline
\end{tabular}

achieved through taking into account LM's prognostic snow in this way without complex changes in the RTTOV code. Presently, clear and cloudy-sky radiance and brightness temperature of Meteosat 7 (two channels, IR and WV) and Meteosat 8 (eight channels) are output variables (see Table 2).

\section{Case study results and discussion}

On 11 September 2003, a well-evolved mesoscale cyclone was situated over Central Europe. Meteosat 7 imagery shows a striking, cyclonically curved cloud pattern in the IR channel (Fig. 1). At 06:00 UTC, a main cloud vortex extends from the center of the cyclone over northern Germany to the German-Polish border, the Czech Republic, Bavaria, Switzerland, Central France to the British Isles. In the wake of the vortex, cold dry air has intruded over the North Sea and the Benelux States to southern Germany and is marked by cloud-free regions or areas with low clouds. Six hours later, the clouds are wound around the quasi-stationary low center above eastern Germany showing a textbook-like vortex. From the northwest, another cloud system enters the domain and covers the British Isles at 12:00 UTC.

All LM experiments (version 3.12) presented in this study are initialized with data of DWD's global model at 11 September 00:00 UTC and use the new prognostic precipitation and cloud ice schemes. The LM experiments differ from each other mainly in the cloud parameters used in RTTOV to compute synthetic satellite imagery (Table 3).

The LM reference experiment na5 uses for RTTOV the model fields cloud liquid water, cloud ice and water content of snow, as forecast by the grid-scale precipitation scheme. The resulting synthetic IR satellite image for 00:00 UTC $+6 \mathrm{~h}$ forecast time is
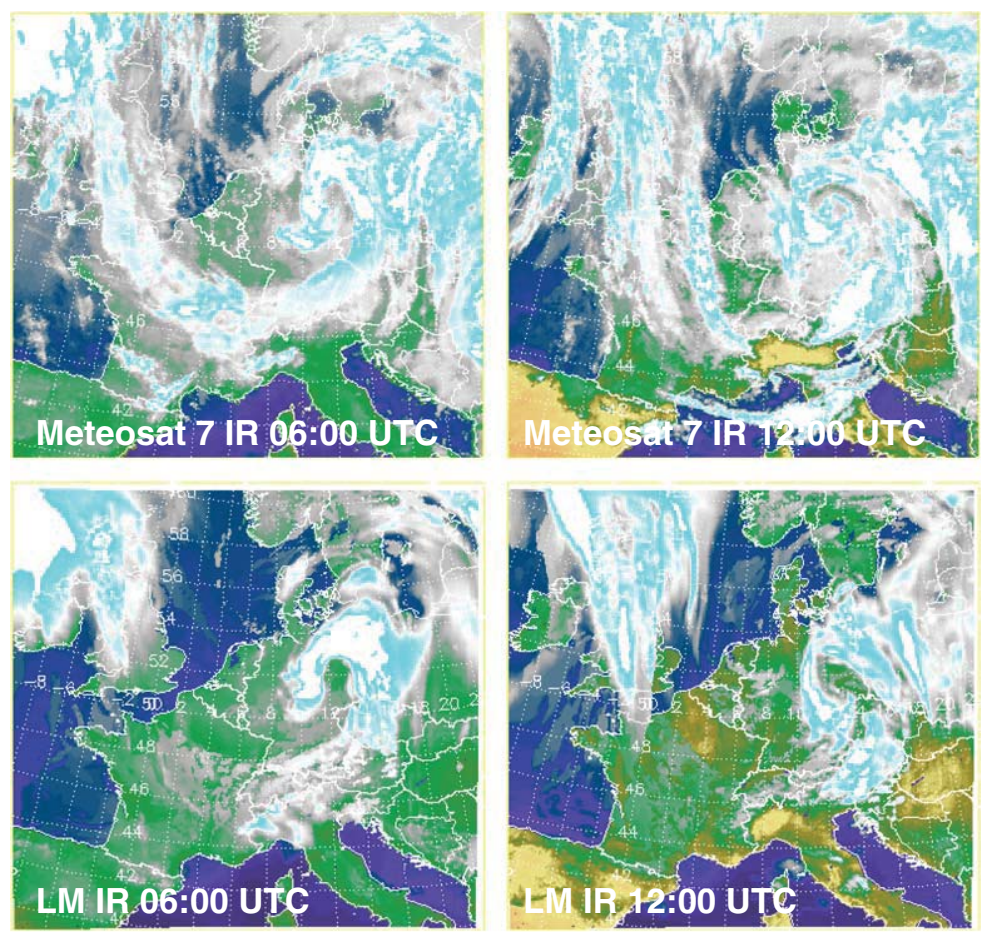

Fig. 1. IR imagery of Meteosat 7 (top) vs. synthetic LM imagery of experiments na5 (bottom) at 06:00 UTC (left) and 12:00 UTC (right) on 11 September 2003. 
Table 3

List of LM experiments for the episode of storm 'Veit'

\begin{tabular}{|c|c|c|c|}
\hline $\begin{array}{l}\text { LM } \\
\text { exp. }\end{array}$ & $\begin{array}{l}\text { Liquid/solid water conc. } \\
\text { profile used in RTTOV }\end{array}$ & $\begin{array}{l}\text { cloud liquid water } \\
\text { used in RTTOV }\end{array}$ & $\begin{array}{l}\text { Critical ice } \\
\text { mixing ratio }\end{array}$ \\
\hline na & qc, qi, qs & None & $0 \mathrm{~kg} / \mathrm{kg}^{-1}$ \\
\hline & $\mathrm{qc}, \mathrm{qi}, \mathrm{qs}$ & Yes & $0 \mathrm{~kg} / \mathrm{kg}^{-1}$ \\
\hline & qc, qi & None & $0 \mathrm{~kg} / \mathrm{kg}^{-1}$ \\
\hline$n a$ & qc, qi, qs & None & $2 \times 10^{-5} \mathrm{~kg} / \mathrm{kg}^{-1}$ \\
\hline
\end{tabular}

Cloud properties forecast by the grid-scale precipitation scheme are cloud liquid water (qc), cloud ice (qi) and water content of snow (qs). The critical ice mixing ratio governs the autoconversion process diminishing cloud ice into precipitating snow.

displayed in Fig. 1, along with the observed images. The key feature of the storm, the cloud vortex across Central Europe, is correctly forecast in position and strength. However, the LM underestimates the amount of cloud. In particular, the trailing cloud band across France as observed by Meteosat 7 is largely missing in the LM forecast: at 06:00 UTC, only low clouds are present, while hardly any clouds appear across France $6 \mathrm{~h}$ later. At 12:00 UTC, the observed clouds across Italy and the Mediterranean and across Ireland are absent in the synthetic imagery. The mismatch of clouds across Ireland and the significant cloud underestimation across Poland can be attributed to the nearby model boundary and associated influences of prescribed input from the global model at the inflow region.

The general underestimation of clouds in LM is evident in histograms of observed and simulated BT. The normalized frequency distribution of BT is displayed for the IR channel comparing Meteosat 7 and LM at 06:00 UTC (Fig. 2). Low BT corresponds to radiation coming from the upper troposphere, while high BT marks the portion emitted by clouds in the lower troposphere or the ground. The IR histogram shows a warm bias of the LM image with BT peaking at $9{ }^{\circ} \mathrm{C}$, i.e. roughly $3 \mathrm{~K}$ warmer than Meteosat 7. The cloud-free and 'low' cloudiness regions $\left(\mathrm{BT}>0{ }^{\circ} \mathrm{C}\right)$ dominate the $\mathrm{LM}$ forecast, which is already apparent in the visual comparison of observed and synthetic satellite imagery in Fig. 1, resulting in the warm bias. The occurrence of clouds attaining BT between $0{ }^{\circ} \mathrm{C}$ and $-20{ }^{\circ} \mathrm{C}$ (mostly 'mid-level' clouds) is underestimated, whereas clouds with BT less than $-20{ }^{\circ} \mathrm{C}$ (mostly 'high' clouds) are well forecast by LM. Hence, the key feature (the comma like vortex of clouds) is captured by LM, but there are problems with forecasting 'mid-level' $\left(-20{ }^{\circ} \mathrm{C}<\mathrm{BT}<0{ }^{\circ} \mathrm{C}\right)$ and 'low' clouds or surface properties $\left(\mathrm{BT}>0{ }^{\circ} \mathrm{C}\right)$ correctly.
A series of LM experiments has been carried out to investigate the sensitivity of synthetic satellite images to LM input fields. In the LM, moist convection is parameterized at $7 \mathrm{~km}$ horizontal resolution following Tiedtke (1989). In this scheme, the diagnostic quantity convective cloud liquid water is used to determine the convective precipitation and is subsequently detrained. In LMSynSat, an option has been provided to use the convective cloud fraction and cloud liquid water in addition to grid-scale cloud liquid water in the RTTOV calculation of BT. In Fig. 2, the effect of this LM experiment na6 is shown. The amount of cloud is slightly increased but the impact is only minor. The repeated detrainment of convective cloud liquid water inhibits the formation of coherent convective cloud structures and leads to a variable, spotty distribution of convection in the synthetic satellite imagery (not shown).

In earlier LM experiments of mid-latitude winter storms using the diagnostic precipitation scheme, Keil et al. (2003) demonstrated the strong sensitivity of high clouds to changes in the cloud microphysics scheme. Non-precipitating cloud-ice is mainly diminished by the autoconversion process, which converts cloud-ice to precipitating snow when a certain cloud-ice threshold, the critical ice mixing ratio, is reached (Lin et al., 1983). For mid-latitude cyclones, Chaboureau et al. (2002) and Keil et al. (2003) found a profound impact of modifications of the ice-to-snow autoconversion threshold on forecast BT. A higher autoconversion threshold implies higher (non-precipitating) cloud-ice contents for

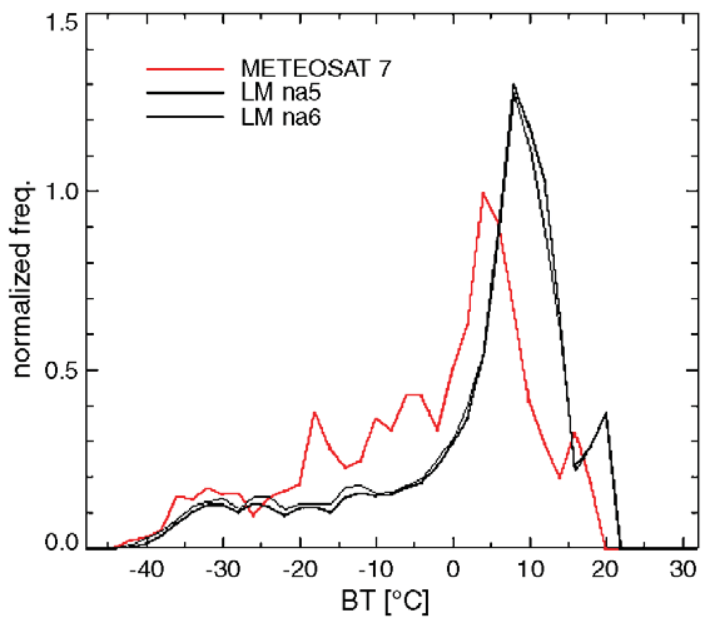

Fig. 2. Histogram of Meteosat 7 (gray) and two LM experiments (na5 and na6; black) in the IR channel at 06:00 UTC 11 September 2003. The histogram is normalized with BT observations. 


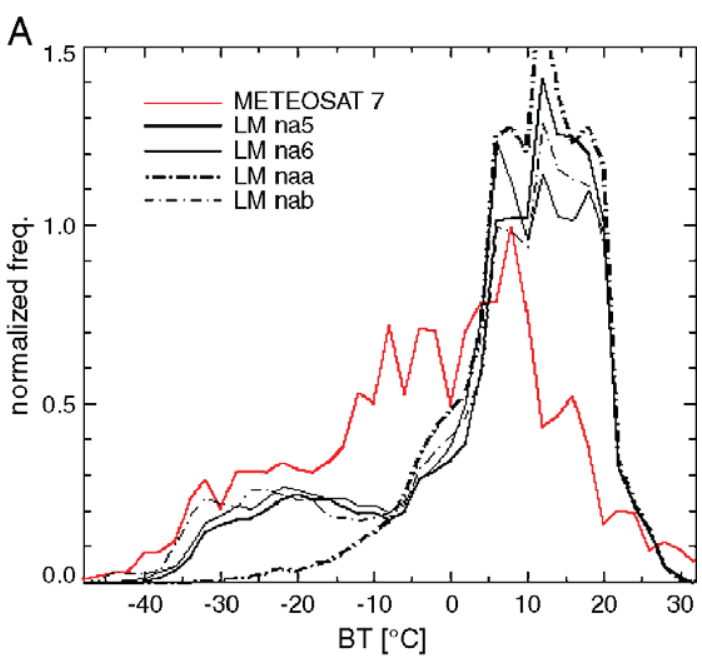

Meteosat(red), LM(black), at $2003091100+12 \mathrm{hrs}$

B

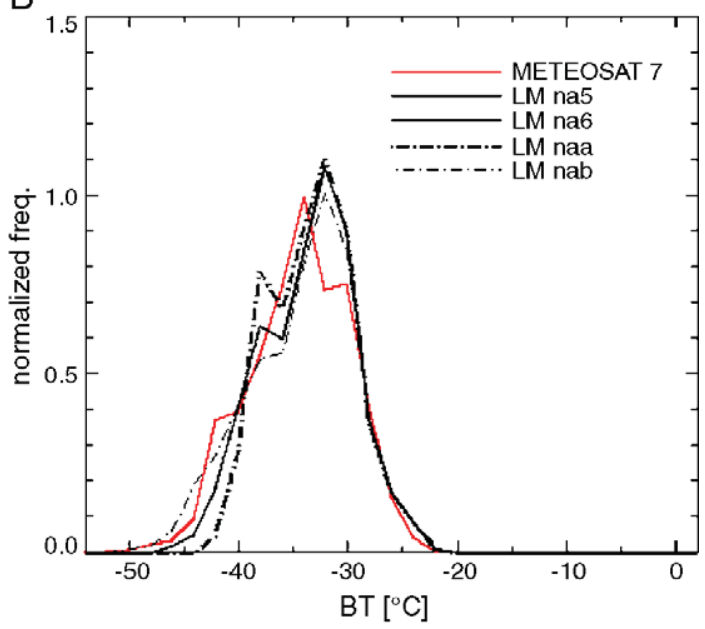

Fig. 3. Histogram of Meteosat 7 (gray) and different LM experiments (black) in the IR (left) and WV (right) channel at 12:00 UTC 11 September 2003. The histogram is normalized with BT observations.

which the resident time is longer than for (precipitating) snow.

The impact of changing the critical ice mixing ratio (to $2 \times 10^{-5} \mathrm{~kg} / \mathrm{kg}$, see Table 3) in conjunction with the new prognostic precipitation scheme that provides the water content of snow as a prognostic variable, is investigated in LM experiment nab. Additionally, the neglect of the water content of snow, i.e. the precipitating snow, in the RTTOV calculation is studied in LM experiment naa (see Table 3). This experiment mimics earlier LM experiments of mid-latitude winter storms using the (grid-scale) diagnostic precipitation scheme (Keil et al., 2003).

In all LM experiments, the location of the cloud vortex is similar to the reference LM experiment resulting from the strong dynamical organization of the flow (not shown). However, the amount of cloud is different and is displayed in the histogram, that is normalized with satellite observations (Fig. 3). All LM experiments have a warm bias overestimating BT larger than $0{ }^{\circ} \mathrm{C}$. In $n a b$, the simulated amount of cloud ice is significantly increased compared to na5. However, the occurrence of high cloudiness in LMSynSat is only slightly increased since already taking into account the prognostic snow (na5 compared to $n a b$ ) remedies a significant amount of the underestimate of high cloudiness found in naa. The use of precipitating snow leads to a significant increase of high cloudiness in LMSynSat, whereas the additional increase in cloud ice caused by changes in the autoconversion process contributes only a little to the total amount of high cloudiness. In contrast to the IR channel, the WV absorption band detects the water vapor content in the upper troposphere (in case no deep opaque cloud is present). This distribution is well captured by the LM and not sensitive to the discussed variations. The WV histogram peaks between $-30{ }^{\circ} \mathrm{C}$ and $-35{ }^{\circ} \mathrm{C}$ with fairly steep flanks (Fig. 3).

Comparison of the correlation between observed and model-simulated BT confirms the similar quality of LM experiments na5, na6 and $n a b$ (see Table 4). Only LM experiment naa (ignoring precipitating snow in RTTOV) has a clearly lower correlation. The temporal evolution of the correlation could serve as a first estimate of the forecast quality of the model. The time series of the correlation between observed and model-simulated BT (in the IR and averaged over the entire model domain) shows variations with best correlation in the morning and worst shortly after noon (Fig. 4). One reason for this behaviour is the well-known difficulty at forecasting surface properties like skin temperature correctly. Moreover, the average correlation values do not provide information about errors in position. Slight spatial and temporal displacements of observed and

Table 4

Daily mean correlation between observed and simulated brightness temperatures of different LM experiments

\begin{tabular}{lll}
\hline $\mathrm{LM}$ & \multicolumn{2}{l}{ Mean correlation } \\
\cline { 2 - 3 } exp. & $\mathrm{BT}_{\mathrm{IR}}$ & $\mathrm{BT}_{\mathrm{WV}}$ \\
\hline na5 & 0.55 & 0.39 \\
$n a 6$ & 0.56 & 0.39 \\
$n a a$ & 0.47 & 0.38 \\
nab & 0.56 & 0.41 \\
\hline
\end{tabular}




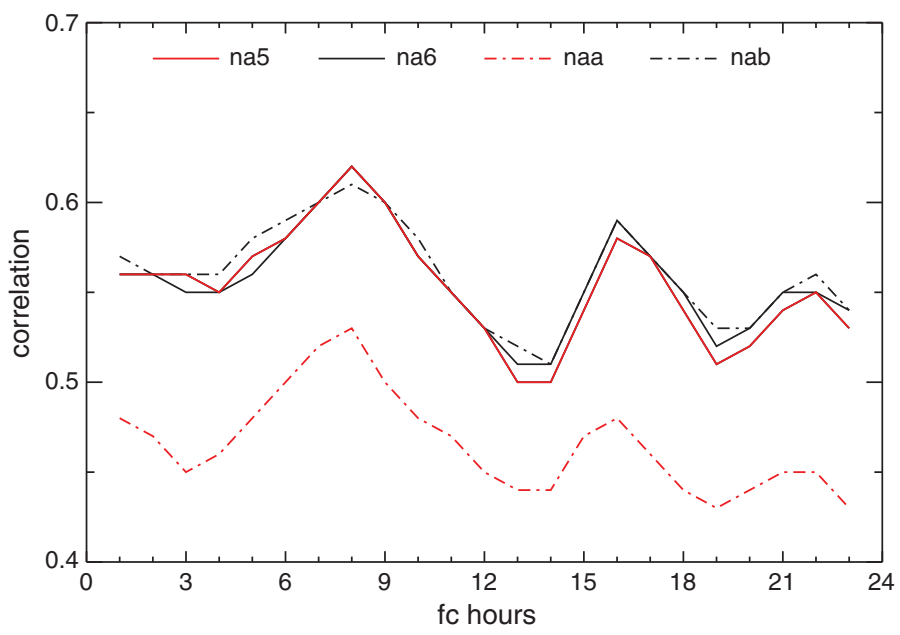

Fig. 4. Time series of correlation of IR Meteosat 7 and synthetic brightness temperature of different LM experiments on 11 September 2003.

model-simulated cloud structures can lead to significant deviations.

\section{Summary}

The new diagnostic LM option (LMSynSat) for generating synthetic satellite imagery allows direct comparison of model forecasts with satellite observations. LMSynSat produces operationally model forecast BT for Meteosat 7 and Meteosat 8 archived in standard GRIB format. The new prognostic precipitation and cloud-ice schemes seem to enable a more realistic representation of cloud properties in LM for use in RTTOV. The present case-study-type investigation indicates that the inclusion of convectively generated cloud water only weakly changes the model-simulated BT, whereas the use of precipitating snow significantly improves the representation of upper tropospheric clouds. With the inclusion of precipitating snow, the modification of the critical ice mixing ratio in the autoconversion process for more realistic representation of upper tropospheric clouds has an only minor effect. The operational production and archiving of synthetic BT will allow systematic investigations of these and other aspects of the representation of clouds in the LM.

A realistic representation of clouds is an essential requirement for a further objective usage of satellite imagery to evaluate forecast quality. There is ongoing work at DLR combining a regional ensemble system COSMO-LEPS (Molteni et al., 2001; Marsigli et al., 2001), synthetic satellite imagery and remote sensing data to develop a probabilistic forecasting system.
Using a pattern recognition algorithm, observed and forecast satellite imagery are exploited to determine realistic members out of an ensemble of forecasts.

\section{Acknowledgements}

Meteosat data are copyrighted by EUMETSAT. The authors thank Ulrich Schättler (DWD) for his support implementing LMSynSat into the LM code.

\section{References}

Baldauf, M., Schulz, J.-P., 2004. Prognostic precipitation in the Lokal-Modell of DWD. COSMO Newsl. (4), 177-180 (http:// cosmomodel.cscs.ch/public/various/newsLetters/newsLetter04).

Chaboureau, J.-P., Cammas, J.-P., Mascart, P., Pinty, J.-P., Claud, C., Roca, R., Morcrette, J.-J., 2000. Evaluation of a cloud system lifecycle simulated by Meso-NH model during FASTEX using METEOSAT radiances and TOVS-3I cloud retrievals. Q. J. R. Meteorol. Soc. 126, 1735-1750.

Chaboureau, J.-P., Cammas, J.-P., Mascart, P., Pinty, J.-P., 2002. Mesoscale model cloud scheme assessment using satellite observations. J. Geophys. Res. 107. doi:10.1029/2001JD000714.

Chevallier, F., Kelly, G., 2002. Model clouds as seen from space: comparison with geostationary imagery in the 11 microns window channel. Mon. Weather Rev. 130, 712-722.

Chevallier, F., Bauer, P., Kelly, G., Jakob, C., McNally, T., 2001. Model clouds over oceans as seen from space: comparison with HIRS/2 and MSU radiance. J. Climate 14, 4216-4229.

Doms, G., Förstner, J., 2004. Development of a kilometer-scale NWP system: LMK. COSMO Newsl. (4), 159-167 (http://cosmomodel. cscs.ch/public/various/newsLetters/newsLetter04).

Doms, G., Schättler, U., 1999. The nonhydrostatic limited-area model LM (Lokal-Modell) of DWD. Tech. rep. Deutscher Wetterdienst, Offenbach am Main. 172 pp.

Doms, G., Majewski, D., Müller, A., Ritter, B., 2004. Recent changes in the cloud-ice scheme. COSMO Newsl. (4), 181-188 (http:// cosmomodel. cscs.ch/public/various/newsLetters/newsLetter04). 
Eyre, J.R., 1991. A fast radiative transfer model for satellite sounding systems. ECMWF, Technical Memorandum, vol. 176. Available from ECMWF, Shinfield Park, Reading RG2 9AX, UK.

Keil, C., Tafferner, A., 2003. LMSynSat Users Guide7pp., available from DLR.

Keil, C., Tafferner, A., Schättler, U., 2003. Evaluating high-resolution model forecasts of European winter storms by use of satellite and radar observations. Weather Forecast. 18, 732-747.

Köpken, C., 2001. Monitoring of METEOSAT WV radiances and solar stray light effects. EUMETSAT/ECMWF Fellowship Programme. Research Report, vol. 1046. Available from ECMWF. Shinfield Park, Reading RG2 9AX, UK.

Lin, Y.-L., Farley, R.D., Orville, H.D., 1983. Bulk parameterization of snow field in a cloud model. J. Clim. Appl. Meteorol. 22, $1065-1092$.

Marsigli, C., Montani, A., Nerozzi, F., Paccagnella, T., Tibaldi, S., Molteni, F., Buizza, R., 2001. A strategy for high-resolution ensemble prediction: Part II. Limited-area experiments in four Alpine flood events. Q. J. R. Meteorol. Soc. 127, 2095-2115.

Matricardi, M., Chevallier, F., Tjemkes, S., 2001. An improved general fast radiative transfer model for the assimilation of radiance observations. ECMWF, Technical Memorandum, vol. 34540. Available from ECMWF. Shinfield Park, Reading RG2 9AX, UK.

Molteni, F., Buizza, R., Marsigli, C., Montani, A., Nerozzi, F., Paccagnella, T., 2001. A strategy for high-resolution ensemble prediction: Part I. Definition of representative members and globalmodel experiments. Q. J. R. Meteorol. Soc. 127, 2069-2094.

Morcrette, J.-J., 1991. Evaluation of model-generated cloudiness: satellite-observed and model-generated diurnal variability of brightness temperature. Mon. Weather Rev. 119, 1205-1224.

Rossow, W.B., Schiffer, R.A., 1991. ISCCP cloud data products. Bull. Am. Meteorol. Soc. 72, 2-20.

Saunders, R., Matricardi, M., Brunel, P., 1999. An improved radiative transfer model for assimilation of satellite radiance observations. Q. J. R. Meteorol. Soc. 125, 1407-1425.

Schmetz, J., van de Berg, L., 1994. Upper tropospheric humidity observations from METEOSAT compared with short term forecast fields. Geophys. Res. Lett. 21, 573-576.

Steppeler, J., Doms, G., Schättler, U., Bitzer, H.W., Gassmann, A., Damrath, U., Gregoric, G., 2003. Meso-gamma scale forecasts using the nonhydrostatic model LM. Meteorol. Atmos. Phys. 82, $75-96$.

Stubenrauch, C.J., Rossow, W.B., Cheruy, F., Chedin, A., Scott, N.A., 1999. Clouds as seen by satellite sounders (3I) and images (ISCCP): Part I. Evaluation of cloud parameters. J. Climate 12, 2189-2213.

Tiedtke, M., 1989. A comprehensive mass flux scheme for cumulus parameterization in large-scale models. Mon. Weather Rev. 117, 1779-1800. 